\title{
Characteristic Functional of a Probability Measure Absolutely Continuous with Respect to a Gaussian Radon Measure
}

\author{
HIRoshi SATo \\ Department of Mathematics, Kyushu University - 33, Hakozaki, Fukuoka, \\ 812 Japan, and Department of Statistics, \\ University of North Carolina, Chapel Hill, North Carolina 27514 \\ Communicated by L. Gross \\ Received August 16, 1984
}

\begin{abstract}
Let $\mu$ and $\mu_{1}$ be probability measures on a locally convex Hausdorff real topological linear space E. C. R. Baker (Lecture Notes in Mathematics No. 109, pp. 33-44, Springer-Verlag, Berlin/New York, 1979) posed the problem of characterizing the absolute continuity of $\mu$ and $\mu_{1}$ by their characteristic functionals. The aim of this paper is to give an answer to this problem in the case where $\mu$ is a Gaussian Radon measure. A Fourier transform shall be defined, the inversion formula established, and then a necessary and sufficient condition given for $\mu_{1}$ to be absolutely continuous with respect to $\mu$ based on the characteristic functional. As applications, for the convolution $\mu_{1}=\mu * v$, where $v$ is a Radon measure on $E$, we shall give some concrete sufficient conditions on $v$ for $\mu * v \ll \mu$. (C) 1985 Academic Press, Inc.
\end{abstract}

\section{INTRODUCTION AND NOTATIONS}

Let $E$ be a locally convex Hausdorff real topological linear space, $E^{*}$ be the topological dual space of $E,\langle x, \xi\rangle$ be the canonical bilinear form on $E \times E^{*}, \mathscr{B}(E)$ be the Borel field of $E$, and $\mathscr{C}\left(E, E^{*}\right)$ be the $\sigma$-algebra generated by $\left\{\langle x, \xi\rangle ; \xi \in E^{*}\right\}$. Every Radon measure $\mu$ on $(E, \mathscr{B}(E))$ is uniquely determined by the characteristic functional

$$
\tilde{\mu}(\xi)=\int_{E} e^{i\langle x, \xi\rangle} d \mu(x), \quad \xi \in E^{*} .
$$

Therefore a Radon measure $\mu_{1}$ is absolutely continuous with respect to $\mu$ (denoted by $\left.\mu_{1} \ll \mu\right)$ on $(E, \mathscr{B}(E))$ if and only if $\mu_{1} \ll \mu$ on $\left(E, \mathscr{C}\left(E, E^{*}\right)\right)$. If $\mu_{1}$ and $\mu$ are mutually absolutely continuous, we denote it by $\mu_{1} \sim \mu$.

Let $\mu$ be a Gaussian Radon measure on $(E, \mathscr{B}(E))$. Then without loss of generality we may assume that the mean is zero (C. Borell [3]) and $E$ is 
the minimal closed linear subspace of probability 1 . Let $H$ be the reproducing kernel Hilbert space of $\mu$ with norm $|\xi|$ and inner product $(\xi, \eta)$. Then $H$ is separable (H. Sato and Y. Okazaki [7], C. Borell [3]), and, identifying $H^{*}$ with $H$, we have $E^{*} \subset H \subset E$, where $E^{*}$ is dense in $H$. The characteristic functional of $\mu$ is $\tilde{\mu}(\xi)=\exp \left[-\frac{1}{2}|\xi|^{2}\right]$.

A subset $A$ of $E$ is called full iff $A \in \mathscr{B}(E)$ and $\mu(A)=1$. A random linear map $T$ of $E$ to a topological linear space $(F, \mathscr{B}(F))$ is a measurable linear map defined on a full linear subspace of $E$. Every random linear map $T$ has an explicit value on $H$ since $H$ is included in every full linear subspace of $E$ (H. Sato and Y. Okazaki [7]). A real-valued random linear map is called a random linear functional. In Section 2 we shall show that every $\xi \in H$ is extended to a random linear functional denoted by $(x, \xi)$ and that every finite-dimensional orthogonal projection $P$ of $H$ is extended to a random linear map of $E$ onto $P H$, which is again denoted by $P$.

The collection of all finite-dimensional orthogonal projections of $H$ is denoted by $\mathscr{P}$. A sequence $\left\{P_{n}\right\}$ in $\mathscr{P}$ is called an approximation sequence iff $x=\lim _{n} P_{n} x$ for every $x \in H$ and $P_{n} H \subset P_{n+1} H, n \in N$.

The characteristic functional of a Radon measure $\mu_{1}$ on $E$ is called $\mu$-continuous iff $\mu_{1} \ll \mu$, and $\mu$-equivalent iff $\mu_{1} \sim \mu$. Let $\tilde{\mu}_{1}(\xi)$ be a $\mu$-continuous characteristic functional. Then there exists the Radon-Nikodym derivative $p(x)=\left(d \mu_{1} / d \mu\right)(x)$, a.e. $(\mu)$, and we have

$$
\tilde{\mu}_{1}(\xi)=\mathbb{E}_{x}\left[e^{i(x, \xi)} p(x)\right], \quad \xi \in H,
$$

where $\mathbb{E}_{x}[]$ is the mathematical expectation with respect to $\mu$ in the variable $x$. Therefore our problem is reduced to characterize the image of

$$
L_{+}^{1}(\mu)=\left\{\phi \in L^{1}(\mu) ; \phi(x) \geqslant 0, \text { a.s. }(\mu)\right\}
$$

under the above transformation. However, it is more convenient for our purpose to define the Fourier transform $\mathscr{F}$ on $L^{1}(\mu)$ by

$$
\widehat{\phi}(\xi)=(\mathscr{F} \phi)(\xi)=e^{(1 / 2)|\xi|^{2}} \mathbb{E}_{x}\left[e^{i(x, \xi)} \phi(x)\right], \quad \xi \in H, \phi \in L^{1}(\mu) .
$$

Then our problem is equivalent to charactcrizing the image of $L_{+}^{1}(\mu)$ under $\mathscr{F}$.

In Section 2 of this paper we prove the Riemann-Lebesgue property for a $\mu$-continuous characteristic functional. In Section 3 we discuss the tame functionals. In Section 4, combining the idea of the "measurable function" of L. Gross [4] and the theory of the classical Fourier transform, we establish the inversion formula for $\mathscr{F}$ (Theorem 4), under the assumption of the integrability, give a necessary and sufficient condition for a continuous functional on $H$ to be the Fourier image of a $\mu$-integrable functional (Theorem 5), give a necessary and sufficient condition for a 
functional on $H$ to be a $\mu$-continuous characteristic function (Theorem 6), and then give an application to the Wiener measure (Theorem 7). In Section 5 we discuss the convolution $\mu_{1}=\mu * v$, where $v$ is a Radon measure on $E$. Since $\mu$ is $H$-quasi-invariant, $\mu * v \ll \mu$ if $v(H)=1$. We give some concrete sufficient conditions on $v$ for $\mu * v \ll \mu$, including the case $v(H)=0$.

Throughout the paper every non-negative definite functional $f$ is assumed to be normalized, i.e., $f(0)=1$, except for Lemmas 3 and 4 , and every Radon measure is a probability measure. The measure $d \lambda(t)$ on $\mathbb{R}^{d}$ denotes the modified Lebesgue measure

$$
d \lambda(t)=d \lambda\left(t_{1}, t_{2}, \ldots t_{d}\right)=(2 \pi)^{-d / 2} d t_{1} d t_{2} \cdots d t_{d} .
$$

We also use the following notations.

$$
\begin{aligned}
C(\xi) & =\exp \left[-\frac{1}{2}|\xi|^{2}\right], \quad \bar{C}(\xi)=\exp \left[\frac{1}{2}|\xi|^{2}\right], \\
\mathbb{R} & =\text { real numbers, } \quad \mathbb{C}=\text { complex numbers, } \\
\mathbb{R}^{d} & =d \text {-dimensional Euclidean space, } \\
\mathbb{N} & =\text { natural numbers, } \quad \mathbb{N}_{0}=\text { non-negative integers } .
\end{aligned}
$$

\section{Riemann-Lebesgue Property}

Let $\mu_{1}$ be a Radon measure on $E$ and $\tau\left(\mu_{1}\right)$ be the topology on $E^{*}$ induced by the convergence in probability $\mu_{1}$. Then, if $\mu_{1} \ll \mu, \tau\left(\mu_{1}\right)$ is weaker than $\tau(\mu)$. On the other hand $\tau(\mu)$ is equivalent to the topology of $H$. Therefore if $\mu_{1} \ll \mu, \tilde{\mu}_{1}(\xi)$ is continuous in the topology of $H$ so that extended to $H$. In this paper we only consider the non-negative definite continuous functionals on $H$.

For every $\xi$ in $H$, since $E^{*}$ is dense in $H$, there exists a sequence $\left\{\xi_{n}\right\}$ in $E^{*}$ such that $\lim _{n}\left|\xi-\xi_{n}\right|=0$. Then, since $\left\{\left\langle x, \xi_{n}\right\rangle\right\} n \in \mathbb{N}$ is a Cauchy sequence in $\tau(\mu),\left\langle x, \xi_{n}\right\rangle$ converges to a real random variable $\xi(x)$ in $\tau(\mu)$. Let $\left\{\left\langle x, \xi_{n_{k}}\right\rangle\right\} k \in \mathbb{N}$ be a subsequence which converges almost surely to $\xi(x)$ and define $(x, \xi)=\lim _{k}\left\langle x, \xi_{n_{k}}\right\rangle$. Then $(x, \xi)$ is a random linear functional on $(E, \mu)$. Therefore the definition of $(x, \xi)$ depends on the choice of the sequence $\left\{\xi_{n_{k}}\right\}$ but it is easy to show that $(x, \xi)$ is defined uniquely up to $\mu$-null sets so that the distribution of $(x, \xi)$ is determined uniquely. $(x, \xi)$ obeys to a Gaussian distribution of mean zero and variance $|\xi|^{2}$ (denoted by $N\left(0,|\xi|^{2}\right)$ ), and $(x, \xi)$ and $(x, \eta), \xi, \eta \in H$, are independent if and only if $(\xi, \eta)=0$.

For every $P$ in $\mathscr{P}$, let $\left\{e_{k}: 1 \leqslant k \leqslant d\right\}$ be a CONS (complete orthonormal system) of $P H$. Then obviously

$$
\stackrel{P}{P} x=\sum_{k=1}^{d}\left(x, e_{k}\right) e_{k}
$$


defines a random linear map of $E$ onto $P H$. The definition of $\stackrel{P}{P}$ depends on the choice of the CONS but is unique up to $\mu$-null sets so that the distribution of $\dot{P} x$ is determined uniquely. We denote $\dot{P}$ again by $P$ and remark that we have

$$
(x, P \xi)=(P x, P \xi)=(P x, \xi)
$$

almost surely on $(E \times E, \mu \times \mu)$.

Let $\mathscr{B}(P)$ be the $\sigma$-algebra on $E$ generated by $\{(x, \xi) ; \xi \in P H\}$, and for every approximation sequence $\left\{P_{n}\right\}$ in $\mathscr{P}$, let $\bigvee_{n} \mathscr{B}\left(P_{n}\right)$ be the $\sigma$-algebra generated by $\bigcup_{n} \mathscr{B}\left(P_{n}\right)$. Then for every $\mathscr{C}\left(E, E^{*}\right)$-measurable function $\phi$ on $E$ there exists a $\bigvee_{n} \mathscr{B}\left(P_{n}\right)$-measurable function $\phi_{1}$ such that $\phi(x)=\phi_{1}(x)$, a.s. $(\mu)$. Therefore it is sufficient to show the absolute continuity on $\bigvee_{n} \mathscr{B}\left(P_{n}\right)$ for an approximation sequence $\left\{P_{n}\right\}$ in $\mathscr{P}$.

For every $\phi$ in $L^{1}(\mu)$ and every $P$ in $\mathscr{P}$ we denote the conditional expectation with respect to $\mathscr{B}(P)$ by

$$
(P \phi)(x)=\mathbb{E}_{x}[\phi \mid \mathscr{B}(P)] .
$$

Let $\left\{e_{k}: 1 \leqslant k \leqslant d\right\}$ be a CONS of $P H$ (so that $d$ is the dimension of $P H$ ). Then there exists a Borel function $(P \phi)^{*}(t)=(P \phi)^{*}\left(t_{1}, t_{2}, \ldots, t_{d}\right)$ on $\mathbb{R}^{d}$ such that

$$
(P \phi)(x)=(P \phi)^{*}\left(\left(x, e_{1}\right),\left(x, e_{2}\right), \ldots,\left(x, e_{d}\right)\right), \quad \text { a.s. }(\mu) .
$$

Theorem 1. Let $\phi$ be in $L^{1}(\mu)$ and define

$$
\widetilde{\phi}(\xi)=\mathbb{E}_{x}\left[e^{i(x, \xi)} \phi(x)\right], \quad \xi \in H .
$$

Then we have the following.

(1) $\bar{\phi}$ is bounded and uniformly continuous on $H$.

(2) For every approximation sequence $\left\{P_{n}\right\}$ in $\mathscr{P}$

$$
\lim _{n} \sup _{\xi \in H}\left|\bar{\phi}(\xi)-\left(\widetilde{P_{n} \phi}\right)(\xi)\right|=0 .
$$

(3) (Riemann-Lebesque property)

$$
\lim _{|\xi| \rightarrow \infty} \tilde{\phi}(\xi)=0
$$

Proof. (1) is trivial.

(2) Let $\left\{P_{n}\right\}$ be an approximation sequence in $\mathscr{P}$. Then 
$P_{n} \phi=\mathbb{E}_{x}\left[\phi \mid \mathscr{B}\left(P_{n}\right)\right]$ converges to $\phi$ in $L^{1}(\mu)$ (J. Neveu [5, Proposition IV2-3]) so that we have

$$
\begin{aligned}
& \sup _{\xi \in H}\left|\tilde{\phi}(\xi)-\left(\widetilde{P_{n} \phi}\right)(\xi)\right| \\
& \quad=\sup _{\xi \in H}\left|\mathbb{E}_{x}\left[e^{i(x, \xi)}\left(\phi(x)-\left(P_{n} \phi\right)(x)\right)\right]\right| \\
& \quad \leqslant \mathbb{E}_{x}\left[\left|\phi(x)-\left(P_{n} \phi\right)(x)\right|\right] \rightarrow 0 \quad \text { as } n \rightarrow+\infty .
\end{aligned}
$$

(3) For every $\varepsilon>0$ by (2) there exists $P$ in $\mathscr{P}$ such that $\sup _{\xi \in H}|\tilde{\phi}(\xi)-\widetilde{(P \phi)}(\xi)|<\frac{1}{2} \varepsilon$. On the other hand we have

$$
\begin{aligned}
|\widetilde{(P \phi)}(\xi)| & =\left|\mathbb{E}_{x}\left[e^{i(x, \xi)}(P \phi)(x)\right]\right| \\
& =\left|\mathbb{E}_{\mathrm{x}}\left[e^{i(x, \xi)}(P \phi)^{*}\left(\left(x, e_{1}\right),\left(x, e_{2}\right), \ldots,\left(x, e_{d}\right)\right)\right]\right| \\
& =C((J-P) \xi) J(\xi), \\
J & =J(\xi)=\int_{R^{d}} e^{i \Sigma_{k} t_{k}\left(\xi, e_{k}\right)}(P \phi)^{*}\left(t_{1}, t_{2}, \ldots, t_{d}\right) C\left(t_{1}, t_{2}, \ldots, t_{d}\right) d \lambda\left(t_{1}, \ldots, t_{d}\right),
\end{aligned}
$$

where $I$ is the identity operator of $H,\left\{e_{k}: 1 \leqslant k \leqslant d\right\}$ is a CONS of $P H$. Then we can easily prove that $\lim _{|\xi| \rightarrow \infty} C((I-P) \xi) J(\xi)=0$ by the classical Riemann-Lebesgue theorem and the boundedness of $C(\xi)$ and $J(\xi)$.

From now on we denote the integral $J(\xi)$ symbolically by

$$
J(\xi)=\int e^{i(P x, P \xi)}(P \phi)(P x) C(P x) d \lambda(P x) .
$$

\section{TAME Functionals}

A $\mu$-measurable functional $\phi=\phi(x)$ on $E$ is called a tame functional if there exists $P$ in $\mathscr{P}$ such that $\phi(x)=\phi(P x)$, a.s. $(\mu)$, and a functional $\psi=\psi(\xi)$ on $H$ is called a tame functional if there exists $P$ in $\mathscr{P}$ such that $\psi(\xi)=\psi(P \xi)$ for every $\xi \in H$. It is obvious that every continuous tame functional on $H$ is extended to a $\mu$-measurable tame functional on $E$.

The following lemma is proved in S. Bochner and K. Chandrasekharan [2, Theorems 37 and 38].

Lemma 1. Define the classical Fourier transform by

$$
f(\alpha)=\int_{R^{d}} e^{i(\alpha, t)} f(t) d \lambda(t), \quad \alpha \in \mathbb{R}^{d},
$$


for $f \in L^{1}\left(\mathbb{R}^{d}, d \lambda\right)$. Then we have

(1) $f(t)=\lim _{\varepsilon \downarrow 0} \int_{R^{d}} e^{-i(t, \alpha)} f(\alpha) C(\sqrt{\varepsilon} \alpha) d \lambda(\alpha)$, a.s. $(d \lambda)$.

(2) In particular if $f(\alpha)$ is d $\lambda(\alpha)$-integrable, then

$$
f(t)=\int_{\mathbb{R}^{d}} e^{-i(t, \alpha)} f(\alpha) d \lambda(\alpha), \quad \text { a.s. }(d \lambda) .
$$

On the other hand we have the following lemma.

Lemma 2. Let $\phi$ be a $\mu$-integrable tame functional on $E$ such that $\phi(x)=\phi(P x)$, a.s. $(\mu)$, for a $P$ in $\mathscr{P}$. Then $\hat{\phi}=\mathscr{F} \phi$ has the representation $\hat{\phi}(\xi)=\hat{\phi}(P \xi)$ for all $\xi$ in $H$, and extends to a tame functional on $E$.

Proof. Assume that $\phi$ satisfies the hypothesis. Then we have

$$
\begin{aligned}
\hat{\phi}(P \xi) & =\bar{C}(P \xi) \mathbb{E}_{x}\left[e^{i(x, P \xi)} \phi(x)\right] \\
& =\bar{C}(P \xi) \mathbb{E}_{x}\left[e^{i(x, P \xi)} \phi(P x)\right] \\
& =\bar{C}(\xi) \mathbb{E}_{x}\left[e^{i(x, \xi)} \phi(P x)\right] \\
& =\bar{C}(\xi) \mathbb{E}_{x}\left[e^{i(x, \xi)} \phi(x)\right]=\hat{\phi}(\xi), \quad \xi \in H .
\end{aligned}
$$

Q.E.D.

For a $\mu$-integrable function $\psi$ we define

$$
(\overline{\mathscr{F}} \psi)(\xi)=\bar{C}(\xi) \mathbb{E}_{x}\left[e^{-i(x, \xi)} \psi(x)\right], \quad \xi \in H .
$$

If $\psi$ is a tame functional, $\overline{\mathrm{F}} \psi$ is also a tame functional on $H$ and can be extended to a tame functional on $E$.

THEOREM 2. Let $\psi$ be a continuous tame functional on $H$ which is $\mu$-integrable on $E$. Then $\psi$ is the Fourier transform of a $\mu$-integrable tame functional on $E$ if and only if $\phi=\overline{\mathscr{F}} \psi$ is $\mu$-integrable.

Moreover if such $\phi$ is $\mu$-integrable, we have $\psi=\mathscr{F} \phi$.

Proof. Assume that $\psi(\xi)=\psi(P \xi), \xi \in H$, for a $P$ in $\mathscr{P}$ and $\phi=\overline{\mathscr{F}} \psi$ is $\mu$-integrable. Then we have

$$
\begin{aligned}
\phi(x) & =\phi(P x) \\
& =\bar{C}(P x) \mathbb{E}_{\xi}\left[e^{-i(\xi, P x)} \psi(\xi)\right] \\
& =\bar{C}(P x) \mathbb{E}_{\xi}\left[e^{-i(P \xi, P x)} \psi(P \xi)\right]
\end{aligned}
$$

so that

$$
\begin{aligned}
C(P x) \phi(P x) & =\mathbb{E}_{\xi}\left[e^{-i(P x, P \xi)} \psi(P \xi)\right] \\
& =\int e^{-i(P x, P \xi)} \psi(P \xi) C(P \xi) d \lambda(P \xi) .
\end{aligned}
$$


This shows that $C(P x) \phi(P x)$ is the classical Fourier inverse transform of $C(P \xi) \psi(P \xi)$. If $\phi(x)=\phi(P x)$ is $\mu$-integrable, then $C(P x) \phi(P x)$ is $d \lambda(P x)$-integrable and we have by Lemma 1(2)

$$
\psi(P \xi) C(P \xi)=\int e^{i(P x, P \xi)} C(P x) \phi(P x) d \lambda(P x), \quad \text { a.s. }(d \lambda),
$$

so that

$$
\begin{aligned}
\psi(\xi) & =\psi(P \xi)=\bar{C}(P \xi) \mathbb{E}_{x}\left[e^{i(P x, P \xi)} \phi(P x)\right] \\
& =\bar{C}(\xi) \mathbb{E}_{x}\left[e^{i(x, \xi)} \phi(P x)\right] \\
& =\bar{C}(\xi) \mathbb{E}_{x}\left[e^{i(x, \xi)} \phi(x)\right]=(\mathscr{F} \phi)(\xi), \quad \text { a.s. }(\mu) .
\end{aligned}
$$

The necessity is a simple corollary of Lemma $1 . \quad$ Q.E.D.

We estimate the Fourier transform of some typical tame functionals.

EXAMPLE 1.

$$
\phi(x) \quad \hat{\phi}(\xi)
$$

(1) $e^{\alpha(x, \eta)}, \quad e^{(1 / 2) \alpha^{2}|\eta|^{2}+\alpha i(\xi, \eta)}, \quad \alpha \in \mathbb{C}, \eta \in H$,

(2) $e^{i(x, \eta)}, \quad e^{-(\xi, \eta)-(1 / 2)|\eta|^{2}}, \quad \eta \in H$,

(3) $(x, \eta)^{n}, \quad i^{n} h_{n}((\xi, \eta)), \quad \eta \in H,|\eta|=1, n \in \mathbb{N}_{0}$,

(4) $h_{n}((x, \eta)), i^{n}(\xi, \eta)^{n}, \quad \eta \in H,|\eta|=1, n \in \mathbb{N}_{0}$,

where $h_{n}(s)=(-1)^{n} e^{s^{2} / 2}\left(d^{n} / d s^{n}\right) e^{-s^{2} / 2}, n \in \mathbb{N}_{0}$.

The proof of the following lemma, which is similar in spirit to that of the Lebesgue differentiability theorems (cf. W. Rudin [6, Theorem 8-6]), was given by $\mathrm{J}$. Bourgain in a personal communication.

LEMMA 3. Let $v$ be a finite measure on $R^{d}$ which is singular to $d \lambda$. Then we have

$$
\lim _{\varepsilon \rightarrow 0}(\varepsilon)^{-d / 2} \int_{\mathbb{R}^{d}} C\left(\frac{s-t}{\sqrt{\varepsilon}}\right) d v(s)=0, \quad \text { a.s. }(d \lambda(t)) .
$$

Proof. Without loss of generality we may assume that $v$ is a probability measure. Since $v$ is singular to $d \lambda$, there exists a Borel subset $N$ of $\mathbb{R}^{d}$ such that $\lambda(N)=0$ and $v(N)=1$.

For every $\varepsilon>0$ define

$$
\begin{aligned}
f_{\varepsilon}(t) & =(\varepsilon)^{-d / 2} \int_{\mathbb{R}^{d}} C\left(\frac{s-t}{\sqrt{\varepsilon}}\right) d v(s) \\
& =(\varepsilon)^{-d / 2} \int_{N} C\left(\frac{s-t}{\sqrt{\varepsilon}}\right) d v(s), \quad t \in \mathbb{R}^{d},
\end{aligned}
$$


and for every $\delta>0$

$$
A=A(\delta)=\left\{t \in \mathbb{R}^{d} ; \limsup _{\varepsilon \rightarrow 0} f_{\varepsilon}(t) \geqslant \delta\right\} .
$$

Then for every $t$ in $A$ there exists a sequence of positive numbers $\varepsilon_{k}=\varepsilon_{k}(t) \rightarrow 0$ and $f_{\varepsilon_{k}}(t) \geqslant \delta$ for all $k \in \mathbb{N}$.

Fix any $t$ in $A$ and any $\varepsilon>0$, define

$$
B_{k}=\left\{s \in \mathbb{R}^{d} ; 2 k \varepsilon \leqslant|s-t|^{2}<2(k+1) \varepsilon\right\}, \quad k \in \mathbb{N}_{0} .
$$

Then we have

$$
\sqrt{\varepsilon^{d}} f_{\varepsilon}(t)=\sum_{k} \int_{B_{k}} C\left(\frac{s-t}{\sqrt{\varepsilon}}\right) d v(s) \geqslant \sqrt{\varepsilon^{d}} \delta
$$

so that

$$
\delta \sqrt{\varepsilon^{d}} \leqslant \sum_{k} e^{-k} v\left(B_{k}\right)
$$

Let $l=l(\varepsilon)$ be the minimal number in $\mathbb{N}_{0}$ greater than $1-\log \delta-(d / 2) \log \varepsilon-\log (e-1) / 2 e$. Then we have

$$
\sum_{k \geqslant l} e^{-k}=\frac{e^{-l}}{1-e^{-1}} \leqslant \frac{\delta}{2} \sqrt{\varepsilon^{d}}
$$

so that

$$
\begin{aligned}
\delta \sqrt{\varepsilon^{d}} & \leqslant \sum_{k} e^{-k} v\left(B_{k}\right) \\
& \leqslant \sum_{k<l} e^{-k} v\left(B_{k}\right)+\sum_{k \geqslant l} e^{-k} \\
& \leqslant \sum_{k<l} e^{-k} v\left(B_{k}\right)+\frac{\delta}{2} \sqrt{\varepsilon^{d}},
\end{aligned}
$$

and

$$
\sum_{k<l} e^{-k} v\left(B_{k}\right) \geqslant \frac{\delta}{2} \sqrt{\varepsilon^{d}}
$$

Define $L=\sqrt{e} /(\sqrt{e}-1)=\sum_{k=0}^{\infty} e^{-k / 2}$ and $M=\delta \sqrt{\varepsilon} / 2 L$. Then there exists $k(\varepsilon) \leqslant l=l(\varepsilon)$ such that $v\left(B_{k(\varepsilon)}\right) \geqslant M e^{k(\varepsilon)}$. In fact assume the contrary. 
Then for every $k \leqslant l$ we have $v\left(B_{k}\right)<M e^{k / 2}$ so that

$$
\begin{aligned}
\sum_{k<l} e^{-k} v\left(B_{k}\right) & <\sum_{k<l} e^{-k / 2} M \\
& <L \frac{\delta \sqrt{\varepsilon^{d}}}{2 L}=\frac{\delta}{2} \sqrt{\varepsilon^{d}},
\end{aligned}
$$

which is a contradiction.

Define for every $k \geqslant 0$

$$
D_{k}=\left\{s \in \mathbb{R}^{d} ;|s-t|^{2} \leqslant 2(k+1) \varepsilon\right\} .
$$

Then we have $\lambda\left(D_{k}\right)=\gamma \sqrt{(k+1) \varepsilon}^{d}$, where $\gamma$ is a positive constant independent of $t, \varepsilon$, and $k$, so that

$$
v\left(D_{k(\varepsilon)}\right) \geqslant v\left(B_{k(\varepsilon)} \geqslant e^{k(\varepsilon) / 2} \frac{\delta}{2 L} \sqrt{\varepsilon^{d}} .\right.
$$

Since

$$
\beta=\inf _{k>0} \frac{e^{k / 2} \delta}{2 L} / \gamma(k+1)^{d / 2}>0
$$

we have

$$
v\left(D_{k(\varepsilon)}\right) \geqslant \beta \gamma \sqrt{(k+1) \varepsilon}=\beta \lambda\left(D_{k(\varepsilon)}\right) .
$$

On the other hand, we have $k(\varepsilon) \leqslant l(\varepsilon)=2-\log \delta-(d / 2) \log \varepsilon-$ $\log (e-1) / 2 e$ so that

$$
\begin{aligned}
\lambda\left(D_{k(\varepsilon)}\right) & =\gamma \sqrt{(k(\varepsilon)+1) \varepsilon}^{d} \\
& \leqslant \gamma \sqrt{(l(\varepsilon)+1) \varepsilon}^{d} \rightarrow 0 \quad \text { as } \varepsilon \rightarrow 0 .
\end{aligned}
$$

Thus we have proved that for every $t$ in $A$ and every $r>0$ there exists a closed ball $D$ with center $t$ and radius less than $r$ such that $v(D) \geqslant \beta \lambda(D)$.

Assume that $b=\lambda(A)>0$. Then there exists a compact subset $K$ of $N$ such that $v(K) \geqslant 1-(\beta / 2) b$ and we have $\lambda(K) \leqslant \lambda(N)=0$. Define $A^{\prime}=A \backslash K$. Then we can choose a sequence $\left\{D_{n}\right\}$ of closed balls such that

$$
\bigcup_{n} D_{n} \supset A^{\prime}, \quad D_{k} \cap K=\varnothing, \quad D_{k} \cap D_{l}=\varnothing(k \neq l), \quad k, l \in \mathbb{N} .
$$

Then we have

$$
\begin{aligned}
v\left(A^{\prime}\right) & =v\left(N \cap \bigcup_{n} D_{n}\right)=v\left(\bigcup_{n} D_{n}\right) \\
& \geqslant \beta \lambda\left(\bigcup_{n} D_{n}\right)=\beta\left\{\lambda\left(\bigcup_{n} D_{n}\right)+\lambda(K)\right\} \\
& \geqslant \beta \lambda(A) \geqslant \beta b .
\end{aligned}
$$


On the other hand, by the assumption we have $v(K) \geqslant 1-\frac{1}{2} \beta b$ so that

$$
\begin{aligned}
v(A) & =v\left(A^{\prime}\right)+v(K) \\
& \geqslant \beta b+1-\frac{1}{2} \beta b \\
& =1+\frac{1}{2} \beta b>1 .
\end{aligned}
$$

This is a contradiction.

Thus we have proved that $\lambda(A(\delta))=0$ for every $\delta>0$, which implies

$$
\lambda\left(t \in \mathbb{R}^{d}: \lim _{\varepsilon} \sup _{\varepsilon}(t)>0\right)=0 .
$$

As an application of Lemma 3 we have the following lemma.

LEMMA 4. Let $f=f(\alpha)$ be a complex-valued function on $\mathbb{R}^{d}$. Then there exists a non-negative $\lambda$-integrable function $p$ on $\mathbb{R}^{d}$ such that

$$
f(\alpha)=\int_{\mathbb{R}^{d}} e^{i(t, \alpha)} p(t) d \lambda(t), \quad \alpha \in \mathbb{R}^{d},
$$

if and only if $f$ is non-negative definite continuous and

$$
f(0)=\int_{\mathbb{R}^{d}} d \lambda(t) \lim _{\varepsilon \rightarrow 0} \int_{\mathbb{R}^{d}} e^{-i(t, \alpha)} f(\alpha) C(\sqrt{\varepsilon \alpha}) d \lambda(\alpha) .
$$

Proof. The necessity is evident from Lemma 1. We shall prove the sufficiency.

Assume that $f$ satisfies the hypothesis. Then by Bochner's theorem there exists a finite measure $m$ on $\mathbb{R}^{d}$ such that

$$
f(\alpha)=\int_{\mathbb{R}^{d}} e^{i(\alpha, t)} d m(t), \quad \alpha \in \mathbb{R}^{d} .
$$

Let $d m(t)=p(t) d \lambda(t)+d v(t)$ be the Lebesgue decomposition of $m$ where $v$ is singular to $d \lambda$. Then we have

$$
f(\alpha)=\int e^{i(\alpha, t)} p(t) d \lambda(t)+\int e^{i(\alpha, t)} d v(t)=f_{1}(\alpha)+f_{2}(\alpha)
$$

and by Lemma 1

$$
p(t)=\lim _{\varepsilon \rightarrow 0} \int e^{i(t, \alpha)} f_{1}(\alpha) C(\sqrt{\varepsilon} \alpha) d \lambda(\alpha), \quad \text { a.s. }(d \lambda(t)) .
$$


On the other hand by Lemma 3 , since $v$ is singular to $\lambda$, we have

$$
\begin{aligned}
& \lim _{\varepsilon \rightarrow 0} \int_{\mathbb{R}^{d}} e^{i(t, \alpha)} f_{2}(\alpha) C(\sqrt{\varepsilon} \alpha) d \lambda(\alpha) \\
& \quad=\lim _{\varepsilon} \int_{\mathbb{R}^{d}} \sqrt{\varepsilon^{-d}} C\left(\frac{s-t}{\sqrt{\varepsilon}}\right) d v(s)=0, \quad \text { a.s. } d \lambda(t) .
\end{aligned}
$$

Thcrefore we have

$$
p(t)=\lim _{\varepsilon} \int e^{i(t, \alpha)} f(\alpha) C(\sqrt{\varepsilon} \alpha) d \lambda(\alpha), \quad \text { a.s. } d \lambda(t) .
$$

Furthermore we have

$$
v\left(\mathbb{R}^{d}\right)+\int_{\mathbb{R}^{d}} p(t) d \lambda(t)=m\left(\mathbb{R}^{d}\right)=f(0)=\int_{\mathbb{R}^{d}} p(t) d \lambda(t) \quad \text { Q.E.D. }
$$

so that $v=0$.

Now we can give a characterization of a $\mu$-continuous characteristic functional in the case of tame functionals.

For a $\mu$-measurable functional $\psi$ on $E$ and $P$ in $\mathscr{P}$ define

$$
\left(\overline{\mathscr{F}}_{P} \psi\right)(x)=\lim _{\varepsilon \rightarrow 0} \bar{C}(P x) \mathbb{E}_{\xi}\left[e^{-i(P x, P \xi)} \psi(P \xi) C(\sqrt{\varepsilon} P \xi)\right]
$$

if it has a meaning. In particular if $\psi(P \xi)$ is $\mu$-integrable, then we have

$$
\left(\overline{\mathscr{F}}_{P} \psi\right)(x)=\bar{C}(P x) \mathbb{E}_{\xi}\left[e^{-i(P x, P \xi)} \psi(P \xi)\right] .
$$

THEOREM 3. Let $\psi$ be a tame functional on $H$ such that $\psi(\xi)=\psi(P \xi)$ for a $P$ in $\mathscr{P}$. Then $f(\xi)=C(\xi) \psi(\xi)$ is a $\mu$-continuous characteristic functional if and only if $f(\xi)$ is non-negative definite continuous on $H$ (so that $\mathscr{F}_{P} \psi$ is well defined $)$ and we have $\mathbb{E}_{x}\left[\left(\overline{\mathscr{F}}_{P} \psi\right)(x)\right]=1$.

And then $\left(\overline{\mathscr{F}}_{P} \psi\right)(x)$ is the Radon-Nikodym derivative.

Proof. Assume that $\psi$ satisfies the hypothesis. Then we have

$$
\begin{aligned}
\left(\overline{\mathscr{F}}_{P} \psi\right)(x) & =\lim _{\varepsilon \rightarrow 0} \bar{C}(P x) \mathbb{E}_{\xi}\left[e^{-i(P x, P \xi)} \psi(P \xi) C(\sqrt{\varepsilon} P \xi)\right] \\
& =\lim _{\varepsilon} \bar{C}(P x) \int e^{-i(P x, P \xi)} \psi(P \xi) C(P \xi) C(\sqrt{\varepsilon P \xi}) d \lambda(P \xi) \\
& =\lim _{\varepsilon} \bar{C}(P x) \int e^{-i(P x, P \xi)} f(P \xi) C(\sqrt{\varepsilon} P \xi) d \lambda(P \xi) .
\end{aligned}
$$


Since $f(P \xi)$ satisfies the hypothesis of Lemma 4 we have

$$
\begin{aligned}
f(P \xi) & =\int e^{i(P x, P \xi)}\left(\overline{\mathscr{F}}_{P} \psi\right)(x) C(P x) d \lambda(P x) \\
& =\mathbb{E}_{x}\left[e^{i(P x, P \xi)}\left(\overline{\mathscr{F}}_{P} \psi\right)(x)\right] .
\end{aligned}
$$

On the other hand by the definition we have $\left(\overline{\mathscr{F}}_{P} \psi\right)(x)=\left(\overline{\mathscr{F}}_{P} \psi\right)(P x)$, a.s. $(\mu)$, so that

$$
\begin{aligned}
f(\xi) & =\bar{C}((I-P) \xi) f(P \xi) \\
& =\bar{C}((I-P) \xi) \mathbb{E}_{x}\left[e^{i(P x, P \xi)}\left(\overline{\mathscr{F}}_{P} \psi\right)(P x)\right] \\
& =\mathbb{E}_{x}\left[e^{i(x, \xi)}\left(\overline{\mathscr{F}}_{P} \psi\right)(x)\right] .
\end{aligned}
$$

The necessity is also proved in the same manner.

Q.E.D.

\section{General Inversion Formula}

THEOREM 4 (Inversion Formula). Let $\phi$ be a $\mu$-integrable function on $E$. Then for an arbitrary approximation sequence $\left\{P_{n}\right\}$ in $\mathscr{P}$ we have

$$
\overline{\mathscr{F}}_{P_{n}} \hat{\phi}=\mathbb{E}_{x}\left[\phi \mid \mathscr{B}\left(P_{n}\right)\right], \quad n \in \mathbb{N} .
$$

Therefore $\overline{\mathscr{F}}_{P_{n}} \hat{\phi}$ converges to $\phi$ simultaneously in $L^{1}(\mu)$ and almost surely.

Proof. By the definition we have

$$
\begin{aligned}
&\left(\overline{\mathscr{F}}_{P_{n}} \hat{\phi}\right)(x) \\
&=\lim _{\varepsilon \rightarrow 0} \bar{C}\left(P_{n} x\right) \mathbb{E}_{\xi}\left[e^{-i\left(P_{n} x, P_{n} \xi\right)} \hat{\phi}\left(P_{n} \xi\right) C\left(\sqrt{\varepsilon} P_{n} \xi\right)\right] \\
&= \lim _{\varepsilon \rightarrow 0} \bar{C}\left(P_{n} x\right) \mathbb{E}_{\xi}\left[e^{-i\left(P_{n} x, P_{n} \xi\right)} \bar{C}\left(P_{n} \xi\right) \mathbb{E}_{y}\left[e^{i\left(y, P_{n} \xi\right)} \phi(y)\right] C\left(\sqrt{\varepsilon} P_{n} \xi\right)\right] \\
&= \lim _{\varepsilon} \bar{C}\left(P_{n} x\right) \mathbb{E}_{\xi}\left[e^{-i\left(P_{n} x, P_{n} \xi\right)} \bar{C}\left(P_{n} \xi\right)\right. \\
&\left.\times \mathbb{E}_{y}\left[e^{i\left(P_{n} y, P_{n} \xi\right)} \mathbb{E}_{y}\left[\phi \mid \mathscr{B}\left(P_{n}\right)\right]\right] C\left(\sqrt{\varepsilon} P_{n} \xi\right)\right] \\
&= \lim _{\varepsilon} \bar{C}\left(P_{n} x\right) \int e^{-i\left(P_{n} x, P_{n} \xi\right)} C\left(\sqrt{\varepsilon} P_{n} \xi\right) d \lambda\left(P_{n} \xi\right) \\
& \times \int e^{i\left(P_{n} y, P_{n} \xi\right)}\left(P_{n} \phi\right)\left(p_{n} y\right) C\left(P_{n} y\right) d \lambda\left(P_{n} y\right) \\
&=\left(P_{n} \phi\right)\left(P_{n} x\right) \\
&= \mathbb{E}_{x}\left[\phi \mid \mathscr{B}\left(P_{n}\right)\right], \quad \text { a.e. }
\end{aligned}
$$

by Lemma 1 .

Q.E.D. 
THEOREM 5. Let $\psi$ be a continuous functional on $H$ such that $\psi\left(P_{n} \xi\right), n \in \mathbb{N}$, are $\mu$-integrable for an approximation sequence $\left\{P_{n}\right\}$ in $\mathscr{P}$. Then there exists a $\mu$-integrable function $\phi$ such that $\psi=\mathscr{F} \phi$ if and only if $\widetilde{\mathscr{F}}_{P_{n}} \psi, n \in \mathbb{N}$, are $\mu$-integrable and converge to $\phi$ in $L^{1}(\mu)$.

Proof. Assume $\psi$ satisfies the hypothesis so that $\overline{\mathscr{F}}_{P_{n}} \psi_{n}$ converges to a $\mu$-integrable function $\phi$ in $L^{1}(\mu)$. Then we have for every $\xi$ in $H$

$$
\begin{aligned}
(\tilde{\mathscr{F}} \phi)(\xi) & =\bar{C}(\xi) \mathbb{E}_{x}\left[e^{i(x, \xi)} \phi(x)\right] \\
& =\lim _{n} \bar{C}(\xi) E_{x}\left[e^{i(x, \xi)}\left(\overline{\mathscr{F}}_{P_{n}} \psi\right)(x)\right] \\
& =\lim _{n} \mathscr{F} \overline{\mathscr{F}}_{P_{n}} \psi .
\end{aligned}
$$

Since $\left(\overline{\mathscr{F}}_{P_{n}} \psi\right)(x)=\left(\overline{\mathscr{F}}_{P_{n}} \psi\right)\left(P_{n} x\right)$, a.s. $(\mu)$, by Theorem 2 we have

$$
\psi\left(P_{n} \xi\right)=\mathscr{F} \overline{\mathscr{F}}_{P_{n}} \psi \rightarrow \psi(\xi) \quad \text { as } n \rightarrow \infty, \xi \in H .
$$

The necessity was proved in Theorem 4.

Q.E.D.

Before stating Lemma 5 , we remark that by the continuous injection of $E$ into $\left(E^{*}\right)^{a}$, the algebraic dual of $E^{*}$ with the weak topology $\sigma\left(\left(E^{*}\right)^{a}, E^{*}\right)$, $\mu$ is extended to a Gaussian Radon measure $\bar{\mu}$ on $\left(E^{*}\right)^{a}$. On the other hand, every non-negative definite continuous functional $f$ on $H$ uniquely defines a probability measure $\bar{v}_{f}$ on $\left(\left(E^{*}\right)^{a}, \mathscr{C}\left(\left(E^{*}\right)^{a}, E^{*}\right)\right)$ such that $f$ is the characteristic function of $\bar{v}_{f}$. Then it is easy to show that $f$ is a $\mu$-continuous characteristic functional on $H$ if and only if $\bar{v}_{f} \ll \bar{\mu}$ on $\left(\left(E^{*}\right)^{a}, \mathscr{C}\left(\left(E^{*}\right)^{a}, E^{*}\right)\right)$.

LEMMA 5. Let $f$ be a non-negative definite continuous functional on $H, \bar{v}_{f}$ be the corresponding probability measure on $\left(\left(E^{*}\right)^{a}, \mathscr{C}\left(\left(E^{*}\right)^{a}, E^{*}\right)\right)$, and $\left\{P_{n}\right\}$ be an arbitrary approximation sequence in $\mathscr{P}$. Then if $\bar{v}_{f}$ is singular to $\bar{\mu}$ on $\mathscr{C}\left(\left(E^{*}\right)^{a}, E^{*}\right)$, then $\left(\overline{\mathscr{F}}_{P_{n}} \psi\right)(x)$ converges to zero almost surely $(\bar{\mu})$, so that almost surely $(\mu)$, where $\psi(\xi)=\bar{C}(\xi) f(\xi), \xi \in H$.

Proof. Since $E$ is a $\bar{\mu}$-measurable full set in $\left(E^{*}\right)^{a}$, random linear maps on $E$ are naturally extended to $\left(E^{*}\right)^{a}$. Denote the $\sigma$-algebra on $\left(E^{*}\right)^{a}$ generated by $\left\{(x, \xi) ; \xi \in P_{n} H\right\}$ by $\overline{\mathscr{B}}_{n}, V_{n} \overline{\mathscr{B}}_{n}$ by $\overline{\mathscr{B}}_{\infty}$, the restriction of $\bar{v}_{f}$ to $\mathscr{B}_{n}$ by $\bar{v}_{n}$, and the restriction of $\bar{\mu}$ to $\overline{\mathscr{B}}_{n}$ by $\bar{\mu}_{n}$, respectively. Then for every $\mathscr{C}\left(\left(E^{*}\right)^{a}, E^{*}\right)$-measurable function $\phi$ on $\left(E^{*}\right)^{a}$ there exists a $\overline{\mathscr{P}}_{\infty}$-measurable function $\phi_{1}$ such that $\phi=\phi_{1}$ a.s. $(\tilde{\mu})$. Let

$$
\bar{v}_{n}(A)=\int_{A} P_{n}(x) d \bar{\mu}_{n}(x)+\bar{\theta}_{n}(A), \quad A \in \overline{\mathscr{B}}_{n}, 1 \leqslant n \leqslant+\infty,
$$

be the Lebesgue decomposition of $\bar{v}_{n}$ with respect to $\bar{\mu}_{n}$ so that $\bar{\theta}_{n}$ is singular to $\bar{\mu}_{n}$. Since $\left\{\overline{\mathscr{B}}_{n}\right\}$ is increasing, by J. Neveu [5, Proposition III- 
2-7] $\left\{p_{n}\right\}$ is a non-negative supermartingale so that $p_{n}$ converges almost surely $(\bar{\mu})$ to $p_{\infty}$. On the other hand $\bar{v}_{f}$ is singular to $\bar{\mu}$ so we have $p_{\infty}=0$, a.s. $(\bar{\mu})$.

On the other hand we have

$$
\begin{aligned}
f\left(P_{n} \xi\right) & =\int e^{i\left(x, P_{n} \xi\right)} p_{n}(x) d \bar{\mu}_{n}(x)+\int e^{i\left(x, P_{n} \xi\right)} d \bar{\theta}_{n}(x) \\
& =f_{1}\left(P_{n} \xi\right)+f_{2}\left(P_{n} \xi\right), \quad \xi \in H,
\end{aligned}
$$

so that by the proof of Lemma 4 we have

$$
\begin{array}{ll}
\overline{\mathscr{F}}_{P_{n}} \psi_{1}=p_{n}(x), & \text { a.s. }\left(\bar{\mu}_{n}\right), \\
\overline{\mathscr{F}}_{P_{n}} \psi_{2}=0, & \text { a.s. }\left(\bar{\mu}_{n}\right),
\end{array}
$$

where we define $\psi_{1}=\bar{C} f_{1}$ and $\psi_{2}=\bar{C} f_{2}$. Therefore we have

$$
\begin{aligned}
\lim _{n} \mathscr{F}_{P_{n}} \psi & =\lim _{n} \overline{\mathscr{F}}_{P_{n}} \psi_{1}+\lim _{n} \overline{\mathscr{F}}_{P_{n}} \psi_{2} \\
& =\lim _{n} p_{n}(x)=0, \quad \text { a.s. }(\bar{\mu}) .
\end{aligned}
$$

THEOREM 6. Let $f$ be a functional on $H$. Then $f$ is a $\mu$-continuous characteristic functional if and only if $f$ is non-negative definite continuous on $H$ and for a (so that any) approximation sequence $P_{n}$ in $\mathscr{P}$

$$
\phi_{n}(x)=\overline{\mathscr{F}}_{P_{n}}(\bar{C} f)(x)
$$

converges in $L^{1}(\mu)$ to $\phi$ such that $\mathbb{E}_{x}[\phi(x)]=1$.

And then $\phi$ is the Radon-Nikodym derivative.

Proof. Assume that $f$ is a $\mu$-continuous characteristic functional with the Radon-Nikodym derivative $p$. Then we have $\bar{C} f=\mathscr{F} p$ so that by Theorem 4

$$
\overline{\mathscr{F}}_{P_{n}}(\bar{C} f)=\mathbb{E}_{x}\left[p \mid \mathscr{B}\left(P_{n}\right)\right]
$$

which converges to $p$ in $L^{1}(\mu)$ as $n \rightarrow \infty$.

Conversely assume that $f$ satisfies the hypothesis of the theorem. Then there exists a probability measure $\bar{v}_{f}$ on $\left(\left(E^{*}\right)^{a}, \mathscr{C}\left(\left(E^{*}\right)^{a}, E^{*}\right)\right)$ with the characteristic functional $f$. Let $d \bar{v}_{f}(x)=p(x) d \bar{\mu}(x)+d \bar{\theta}(x)$ be the Lebesgue decomposition of $\bar{v}_{f}$ with respect to $\bar{\mu}$, the extension of $\mu$ to $\left(E^{*}\right)^{a}$. Then we have

$$
\begin{aligned}
f(\xi) & =\int e^{i(x, \xi)} p(x) d \bar{\mu}(x)+\int e^{i(x, \xi)} d \bar{\theta}(x) \\
& =f_{1}(\xi)+f_{2}(\xi), \quad \xi \in H .
\end{aligned}
$$


By Theorem $4 \overline{\mathscr{F}}_{p_{n}}\left(\bar{C} f_{1}\right)$ converges to $p$ in $L^{1}(\bar{\mu})$. On the other hand, since $\theta$ is singular to $\bar{\mu}$, by Lemma 5 either $\bar{F}_{p_{n}}\left(\bar{C} f_{2}\right)$ converges to zero in $L^{1}(\bar{\mu})$ or does not converge in $L^{1}(\bar{\mu})$. By hypothesis $\overline{\mathscr{F}}_{P_{n}}(\bar{C} f)=\overline{\mathscr{F}}_{P_{n}}\left(C f_{1}\right)+\overline{\mathscr{F}}_{P_{n}}\left(\bar{C} f_{2}\right)$ converges in $L^{1}(\bar{\mu})$ so that converges to $p$ and we have $\left.\int p(x) d \mu(x)=1=\bar{v}_{f}\left(E^{*}\right)^{a}\right)$. Therefore $\theta=0$ and the theorem is proved.

Q.E.D.

Reformulating Theorem 6, we have the following proposition.

PROPOSITION 1. Let $f$ be the characteristic functional of a Radon measure $\mu_{1}$ on $E$. Then $\mu_{1} \ll \mu$ if and only if $f$ is continuous on $H$ and for a (so that any) approximation sequence $\left\{P_{n}\right\}$ in $\mathscr{P}$

$$
\begin{aligned}
\phi_{n}(x)= & \lim _{\varepsilon \rightarrow 0} \varepsilon^{-d(n)} \int_{E} \exp \left[-\frac{1}{2 \varepsilon^{2}}\left|P_{n} x-P_{n} y\right|^{2}+\frac{1}{2}\left|P_{n} x\right|^{2}\right] \\
& \times d \mu_{1}(y), \quad n \in \mathbb{N},
\end{aligned}
$$

where $d(n)$ is the dimension of $P_{n} H$, converges in $L^{1}(\mu)$ to $\phi$ such that $\mathbb{E}_{x}[\phi(x)]=1$.

And then $\phi$ is the Radon-Nikodym derivative.

Proof. Since we have

$$
f(\xi)=\int_{E} e^{i(y, \xi)} d \mu_{1}(y), \quad \xi \in H,
$$

Fubini's theorem implies that

$$
\begin{aligned}
\overline{\mathscr{F}}_{P_{n}} & (\bar{C} f)(x) \\
= & \lim _{\varepsilon \rightarrow 0} \bar{C}\left(P_{n} x\right) \mathbb{E}_{\xi}\left[e^{-i\left(P_{n} x, P_{n} \xi\right)-\left(\varepsilon^{2} / 2\right)\left|P_{n} \xi\right|^{2}} \bar{C}\left(P_{n} \xi\right) f\left(P_{n} \xi\right)\right] \\
= & \lim _{\varepsilon \rightarrow 0} \bar{C}\left(P_{n} x\right) \int e^{-i\left(P_{n} x, P_{n} \xi\right)-\left(\varepsilon^{2} / 2\right)\left|P_{n} \xi\right|^{2}} d \lambda\left(P_{n} \xi\right) \int_{E} e^{i\left(y, P_{n} \xi\right)} d \mu_{1}(y) \\
= & \lim _{\varepsilon \rightarrow 0} \varepsilon^{-d(n)} \int_{E} \exp \left[-\frac{1}{2 \varepsilon^{2}}\left|P_{n} x-P_{n} y\right|^{2}+\frac{1}{2}\left|P_{n} x\right|^{2}\right] d \mu_{1}(y) .
\end{aligned}
$$

Then Theorem 6 proves the proposition.

Q.E.D.

Let $\Delta_{n}=\left\{e_{n, 1}, e_{n, 2}, \ldots, e_{n, d(n)}\right\}, n \in \mathbb{N}$, be a sequence of ONS in $H$. Then $\left\{\Delta_{n}\right\}$ is called an approximation sequence of ONS in $E^{*}$ iff we have $\Delta_{n} \subset E^{*}, \Delta_{n} \subset \Delta_{n+1}, n \in \mathbb{N}$, and the closed linear span of $\cup_{n} \Delta_{n}$ coincides 
with $H$. Let $\left\{\Delta_{n}\right\}$ be an approximation sequence of ONS in $E^{*}$. Then for every $n$ the projection

$$
P_{n} x=\sum_{k=1}^{d(n)}\left\langle x, e_{n, k}\right\rangle e_{n, k}
$$

is well defined for all $x \in E$ and it is easy to show that $\left\{P_{n}\right\}$ is an approximation sequence in $\mathscr{P}$. A slight modification of Proposition 1 gives the following proposition.

Proposition 2. Let $\mu_{1}$ be a Radon measure on $E$. Then $\mu_{1} \ll \mu$ if and only if there exists an approximation sequence of ONS in $E^{*}$, say, $\Delta_{n}=\left\{e_{n, 1}, e_{n, 2}, \ldots, e_{n, d(n)}\right\}, n \in \mathbb{N}$, such that

$$
\begin{aligned}
\phi_{n}(x)= & \lim _{\varepsilon \rightarrow 0} \varepsilon^{-d(n)} \int_{E} \exp \left[-\frac{1}{2 \varepsilon^{2}} \sum_{k=1}^{d(n)}\left\{\left(1-\varepsilon^{2}\right)\left\langle x, e_{n, k}\right\rangle^{2}\right.\right. \\
& \left.\left.-2\left\langle x, e_{n, k}\right\rangle\left\langle y, e_{n, k}\right\rangle+\left\langle y, e_{n, k}\right\rangle^{2}\right\}\right] d \mu_{1}(y)
\end{aligned}
$$

converges in $L^{1}(\mu)$ to $\phi$ such that $\mathbb{E}_{x}[\phi(x)]=1$.

And then $\phi$ is the Radon-Nikodym derivative.

Proof. Let $P_{n}$ be the orthogonal projection of $H$ onto the linear span of $\Delta_{n}, n \in \mathbb{N}$. Then $\left\{P_{n}\right\}$ is an approximation sequence in $\mathscr{P}$ and we have

$$
\begin{aligned}
& -\frac{1}{2 \varepsilon^{2}}\left|P_{n} x-P_{n} y\right|^{2}+\frac{1}{2}\left|P_{n} x\right|^{2} \\
& \quad=\frac{1}{2 \varepsilon^{2}} \sum_{k=1}^{d(n)}\left\{\left(1-\varepsilon^{2}\right)\left\langle x, e_{n, k}\right\rangle^{2}-2\left\langle x, e_{n, k}\right\rangle\left\langle y, e_{n, k}\right\rangle+\left\langle y, e_{n, k}\right\rangle^{2}\right\},
\end{aligned}
$$

which is well defined for all $x, y$ in $E$ since $\Delta_{n} \subset E^{*}, n \in \mathbb{N}$. Therefore, without using the continuity of $\tilde{\mu}_{1}(\xi)$ on $H$, from the hypothesis we can construct the Radon-Nikodym derivative and the sufficiency is proved.

The necessity is proved as a simple corollary of Proposition 1. Q.E.D.

We apply Proposition 2 to the Wiener measure.

Let $B(t), 0 \leqslant t \leqslant 1$, be the standard Brownian motion, $X(t)$ be an arbitrary continuous process on $[0,1]$ vanishing at zero. Then $B(t)$ and $X(t)$ induce the Wiener measure $\mu$ and a Radon measure $\mu_{1}$ on the real separable Banach space $C[0,1]$, the space of all continuous functions vanishing at zero, respectively. The topological dual space of $C[0,1]$ is $\mathscr{M}(0,1]$, the space of all signed measures on $(0,1]$.

Let

$$
\mathscr{D}_{n}: 0=t_{n, 0}<t_{n, 1}<\cdots<t_{n, d(n)}=1
$$

be a decomposition of $[0,1]$ such that $\mathscr{D}_{n+1}$ is a refinement of $\mathscr{D}_{n}, n \in \mathbb{N}$. 
Let $\delta(t)$ be the Dirac measure concentrated on $t[0,1]$,

$$
e_{n, k}=\left|\Delta_{n, k}\right|^{-1 / 2}\left\{\delta\left(t_{n, k}\right)-\delta\left(t_{n, k-1}\right\}, \quad 1 \leqslant k \leqslant d(n), n \in \mathbb{N},\right.
$$

where $\left|\Delta_{n, k}\right|=t_{n, k}-t_{n, k-1}$, and assume that $\lim _{n} \max _{k}\left|\Delta_{n, k}\right|=0$. Then it is not difficult to show that $\Delta_{n}=\left\{e_{n, 1}, e_{n, 2}, \ldots, e_{n, d(n)}\right\}, n \in \mathbb{N}$, is an approximation sequence of ONS in $\mathscr{M}(0,1]$. As a corollary of Proposition 2 we have the following theorem.

THEOREM 7. Let $B(t), 0 \leqslant t \leqslant 1$, be the standard Brownian motion, $X(t)$, $0 \leqslant t \leqslant 1$, be an arbitrary continuous process vanishing at zero, $\mu$ be the Wiener measure induced by $B(t)$, and $\mu_{1}$ be the Radon measure induced by $X(t)$ on $C[0,1]$. Then $\mu_{1} \ll \mu$ if and only if

$$
\begin{aligned}
\phi_{n} & =\lim _{\varepsilon \rightarrow 0} \varepsilon^{-d(n)} \mathbb{E}^{X} \\
& \times\left[\exp -\frac{1}{2 \varepsilon^{2}} \sum_{k=1}^{d(n)} \frac{1}{\left|\Delta_{n, k}\right|}\left\{\left(1-\varepsilon^{2}\right) B\left(\Delta_{n, k}\right)^{2}-2 B\left(\Delta_{n, k}\right) X\left(\Delta_{n, k}\right)+X\left(\Delta_{n, k}\right)^{2}\right\}\right]
\end{aligned}
$$

converges in $L^{1}(\mu)$ to $\phi$ such that $\mathbb{E}^{B}[\phi]=1$, where $B\left(\Delta_{n, k}\right)=$ $B\left(t_{n, k}\right)-B\left(t_{n, k-1}\right), X\left(\Delta_{n, k}\right)=X\left(t_{n, k}\right)-X\left(t_{n, k-1}\right), 1 \leqslant k \leqslant d(n), n \in \mathbb{N}$, and $\mathbb{E}^{X}[]$ and $\mathbb{E}^{B}[]$ are the expectations with respect to $X(t)$ and $B(t)$, respectively.

And then $\phi$ is the Radon-Nikodym derivative.

\section{Convolution}

Let $v$ be a Radon measure on $E$ and $\mu * v$ be the convolution of $\mu$ and $v$. Then the characteristic functional is given by $\widetilde{\mu * v}(\xi)=\tilde{\mu}(\xi) \tilde{v}(\xi)=$ $C(\xi) \tilde{v}(\xi), \xi \in E^{*}$, where $\tilde{v}$ is the characteristic functional of $v$. If $\mu * v \ll \mu$, then $\tilde{v}(\xi)$ is continuous on $H$. The aim of this section is to give some sufficient conditions on $v$ for $\mu * v \ll \mu$.

LemMa 6. (1) If $v=\delta_{x}$, the Dirac measure concentrated on $x \in E$, then $\mu * \delta_{x} \ll \mu$ if and only if $x \in H$.

(2) If $\nu(H)=1$, then $\mu * \nu \ll \mu$.

(3) Assume that $\mu * v \ll \mu$ and let $E_{0}$ be a full linear subspace of $E$. Then we have $v\left(E_{0}\right)=1$.

Proof. (1) is well known. 
(2) Assume that $v(H)=1$ and $\mu(N)=0$. Then by $\mu(N-x)=\left(\mu * \delta_{x}\right)(N)=0$ for all $x \in H$ so that we have

$$
\begin{aligned}
(\mu * v)(N) & =\int_{E} \mu(N-x) d v(x) \\
& =\int_{H} \mu(N-x) d v(x)=0 .
\end{aligned}
$$

(3) Assume that $\mu * v \ll \mu$ and let $E_{0}$ be a full linear subspace. Then we have

$$
1=(\mu * v)\left(E_{0}\right)=\int_{E} \mu\left(E_{0}-x\right) d v(x),
$$

so that $v\left(x \in E: \mu\left(E_{0}-x\right)=1\right)=1$. Since $E_{0}$ is a linear subspace, we have $\left(E_{0}-x\right) \cap E_{0}=\varnothing$ if $x \notin E_{0}$. Therefore we have $\left\{x \in E ; \mu\left(E_{0}-x\right)=1\right\} \subset E_{0}$ and $v\left(E_{0}\right) \geqslant v\left(x \in E: \mu\left(E_{0}-x\right)=1\right)=1$.

By Lemma 6 our interest is reduced to the case where $v(H)=0$.

Since $\tilde{v}$ is bounded, Theorem 5 is applicable and we have the following proposition as a corollary.

Proposition 3. Let $v$ be a Radon measure on $E$. Then we have $\mu * v \ll \mu$ if and only if $\tilde{v}(\xi)$ is continuous on $H$ and there exists an approximation sequence $\left\{P_{n}\right\}$ in $\mathscr{P}$ such that

$$
\phi_{n}(x)=\int_{E} e^{\left(P_{n} x, P_{n} y\right)-(1 / 2)\left|P_{n} y\right|^{2}} d v(y)
$$

converges in $L^{1}(\mu)$.

And then the limit function is the Radon-Nikodym derivative.

Proof. Let $P_{n}$ be an approximation sequence in $\mathscr{P}$. Then, since $\left\{P_{n}\right\}$ is bounded so that integrable for every $n \in \mathbb{N}$, we have

$$
\begin{aligned}
\phi_{n}(x) & =\overline{\mathscr{F}}_{P_{n}} \tilde{v}(x) \\
& =C\left(P_{n} x\right) \mathbb{E}_{\xi}\left[e^{-i\left(P_{n} x, P_{n} \xi\right)} \int_{E} e^{i\left(P_{n} y, P_{n} \xi\right)} d v(y)\right] \\
& =\int_{E} e^{\left(P_{n} x, P_{n} y\right)-(1 / 2)\left|P_{n}, y\right|^{2}} d v(y),
\end{aligned}
$$

which is $\mu$-integrable for $C(\xi) \tilde{v}(\xi)$ is non-negative definite. Therefore Theorem 5 proves the theorem.

Q.E.D. 
As in Proposition 2 we can modify Theorem 7 as follows. The proof is similar to that of Proposition 2.

Proposition 4. Let $v$ be a Radon measure on $E$. Then we have $\mu * v \ll \mu$ if and only if there exists an approximation sequence of ONS in $E^{*}$, say, $\Delta_{n}=\left\{e_{n, 1}, e_{n, 2}, \ldots, e_{n, d(n)}\right\}, n \in \mathbb{N}$, such that

$$
\phi_{n}(x)=\int_{E} \exp \left[\sum_{k=1}^{d(n)}\left\langle x, e_{n, k}\right\rangle\left\langle y, e_{n, k}\right\rangle--\frac{1}{2}\left\langle y, e_{n, k}\right\rangle^{2}\right] d v(y)
$$

converges in $L^{1}(\mu)$.

And then the limit function is the Radon-Nikodym derivative.

Proposition 4 gives the following theorem in a similar manner to the proof of Theorem 7. We use the same notations with Theorem 7.

THEOREM 8. Let $B(t), 0 \leqslant t \leqslant 1$, be the standard Brownian motion, $X(t)$, $0 \leqslant t \leqslant 1$, be an arbitrary continuous process vanishing at 0 and independent of $\{B(t)\}, \mu$ be the Wiener measure induced by $B(t)$, and $v$ be the Radon measure induced by $X(t)$ on $C[0,1]$. Then $B(t)+X(t), 0 \leqslant t \leqslant 1$, induces $\mu * v$ and we have $\mu * v \ll \mu$ if and only if there exists a sequence of decomposition of $[0,1]$

$$
\mathscr{D}_{n}: 0=t_{n, 0}<t_{n, 1}<\cdots<t_{n, k}<\cdots t_{n, d(n)}=1
$$

such that $\mathscr{D}_{n+1}$ is a refinement of $\mathscr{D}_{n}, n \in \mathbb{N}, \lim _{n} \max _{k}\left|\Delta_{n, k}\right|=0$, and

$$
\phi_{n}=\mathbb{E}^{X}\left[\exp \left(\sum_{k=1}^{d(n)} \frac{1}{\left|\Delta_{n, k}\right|}\left\{B\left(\Delta_{n, k}\right) X\left(\Delta_{n, k}\right)-\frac{1}{2} X\left(\Delta_{n, k}\right)^{2}\right\}\right)\right]
$$

converges in $L^{1}(\mu)$.

The following theorems are also applications of Proposition 4.

THEOREM 9. Let $v$ be a Radon measure on $E$ and assume that there exists a CONS $\left\{e_{k}\right\}$ of $H$ chosen from $E^{*}$ such that

$$
\sup _{n} \int_{E \times E} \exp \left(\sum_{k=1}^{n}\left\langle y, e_{k}\right\rangle\left\langle y^{\prime}, e_{k}\right\rangle\right) d v(y) d v\left(y^{\prime}\right)<+\infty .
$$

Then $\mu * v \ll \mu$.

Proof. Obviously $\Delta_{n}=\left\{e_{1}, e_{2}, \ldots, e_{n}\right\}, n \in \mathbb{N}$, is an approximation sequence of ONS in $E^{*}$ so that Proposition 4 is applicable and we have

$$
\phi_{n}(x)=\int_{E} \exp \left(\sum_{k=1}^{n}\left\langle x, e_{k}\right\rangle\left\langle y, e_{k}\right\rangle-\frac{1}{2}\left\langle y, e_{k}\right\rangle^{2}\right) d v(y) .
$$


On the other hand since $\left\{\left\langle x, e_{k}\right\rangle\right\}$ is a standard Gaussian sequence so that $\phi_{n}$ is a $\mathscr{B}\left(P_{n}\right)$-martingale where $P_{n}$ is the orthogonal projection of $H$ to the linear span of $\left\{e_{1}, e_{2}, \ldots, e_{n}\right\}$.

Then

$$
\begin{aligned}
& \sup _{n} \mathbb{E}_{x}\left[\phi_{n}(x)^{2}\right] \\
& \quad=\sup _{n} \int_{E \times E} \exp \left(\sum_{k=1}^{n}\left\langle y, e_{k}\right\rangle\left\langle y^{\prime}, e_{k}\right\rangle\right) d v(y) d v\left(y^{\prime}\right)<+\infty
\end{aligned}
$$

implies the uniform integrability of $\left\{\phi_{n}\right\}$ and $\phi_{n}$ converges in $L^{1}(\mu)$.

Q.E.D.

Before stating Theorem 10 we prove the following lemmas.

LeMmA 7. Let $\left\{X_{k}\right\}$ be an independent real random sequence on $(E, \mu)$ such that the expectation $E_{x}\left[X_{k}(x)\right]=0,1+X_{k}>0$, a.s., $k \in \mathbb{N}$, and $Z=\lim _{n} \prod_{k=1}^{n}\left(1+X_{k}\right)>0$, a.s. Then $Z_{n}=\prod_{k=1}^{n}\left(1+X_{k}\right)$ converges to $Z$ in $L^{1}(\mu)$.

Proof. Define $Y_{k}=\left(1+X_{k}\right)^{1 / 2}, k \in \mathbb{N}$. Then we have $\mathbb{E}_{x}\left[Y_{k}\right] \leqslant 1$ and

$$
\begin{aligned}
\prod_{k=1}^{\infty} \mathbb{E}_{x}\left[Y_{k}\right] & =\lim _{n} \prod_{k=1}^{n} \mathbb{E}_{x}\left[Y_{k}\right] \\
& =\lim _{n} \mathbb{E}_{x}\left[\prod_{k=1}^{n} Y_{k}\right]=\lim _{n} \mathbb{E}_{x}\left[Z_{n}^{1 / 2}\right] \\
& \geqslant \mathbb{E}_{x}\left[\underset{n}{\liminf } Z_{n}^{1 / 2}\right]=\mathbb{E}_{x}\left[Z^{1 / 2}\right]>0 .
\end{aligned}
$$

Therefore we have for every $n, m \in \mathbb{N}(n<m)$

$$
\begin{aligned}
\mathbb{E}_{x}\left[\left|Z_{n}-Z_{m}\right|\right]^{2} & =\mathbb{E}_{x}\left[\left|Z_{n}^{1 / 2}-Z_{m}^{1 / 2}\right|\left|Z_{n}^{1 / 2}+Z_{m}^{1 / 2}\right|\right]^{2} \\
& \leqslant 4 \mathbb{E}_{x}\left[\left|Z_{n}^{1 / 2}-Z_{m}^{1 / 2}\right|^{2}\right] \\
& =8 \mathbb{E}_{x}\left[1-Z_{n}^{1 / 2} Z_{m}^{1 / 2}\right] \\
& =8\left\{1-\mathbb{E}_{x}\left[\prod_{n<k \leqslant m} Y_{k}\right]\right\} \\
& =8\left\{1-\prod_{n<k \leqslant m} \mathbb{E}_{x}\left[Y_{k}\right]\right\} \rightarrow 0 \quad \text { as } n, m \rightarrow \infty . \quad \text { Q.E.D. }
\end{aligned}
$$

LEMMA 8. Let $\left\{x_{k}\right\}$ be a real sequence such that $x_{k}>-1, k \in \mathbb{N}$. If $\sum_{k} x_{k}$ and $\sum_{k} x_{k}^{2}$ converge, then $Z_{n}=\prod_{k=1}^{n}\left(1+x_{k}\right)$ converges to a positive number. 
Proof. Since $\sum x_{k}$ converges, we have $\lim _{k} x_{k}=0$ so that we may assume $\left|x_{k}\right|<1, k \in \mathbb{N}$. By the Taylor expansion we have

$$
\sum_{k} \log \left(1+x_{k}\right)=\sum_{k} \sum_{n=1}^{\infty} \frac{(-1)^{n+1}}{n} x_{k}^{n} .
$$

For every $N, M(N<M) \in \mathbb{N}$ we have

$$
\begin{aligned}
\left|\sum_{N<k<M} \sum_{n \geqslant 2} \frac{(-1)^{n+1}}{n} x_{k}^{n}\right| & \leqslant \sum_{n \geqslant 2} \frac{1}{n} \sum_{N<k<M}\left|x_{k}\right|^{n} \\
& \leqslant \sum_{n \geqslant 2} \frac{1}{n}\left(\sum_{N<k<M} x_{k}^{2}\right)^{n / 2} \rightarrow 0 \quad \text { as } N, M \rightarrow+\infty
\end{aligned}
$$

sincc $\sum_{k} x_{n}^{2}<+\infty$. Therefore

$$
\sum_{k} \log \left(1+x_{k}\right)=\sum_{k} x_{k}+\sum_{n \geqslant 2} \frac{(-1)^{n+1}}{n} \sum_{k} x_{k}^{n}
$$

converges.

Q.E.D.

THEOREM 10. Let $v$ be a Radon measure on $E$ and assume that there exists a CONS $\left\{e_{k}\right\}$ of $H$ chosen from $E^{*}$ such that $\left\{\left\langle y, e_{k}\right\rangle\right\}$ is an independent symmetric random sequence on $(E, v)$, and

$$
M=\sum_{n=2}^{\infty} \frac{1}{2^{n} n !} \sum_{k} \int_{E}\left\langle y, e_{k}\right\rangle^{2 n} e^{-(1 / 2)\left\langle y, e_{k}\right\rangle^{2}} d v(y)<+\infty .
$$

Then we have $\mu * v \sim \mu$.

Proof. Assume that the hypothesis is satisfied. Since $\left\{\left\langle y, e_{k}\right\rangle\right\}$ are independent, we have

$$
\begin{aligned}
\phi_{n}(x) & =\int_{E} \prod_{k=1}^{n} \exp \left[\left\langle x, e_{k}\right\rangle\left\langle y, e_{k}\right\rangle-\frac{1}{2}\left\langle y, e_{k}\right\rangle^{2}\right] d v(y) \\
& =\prod_{k=1}^{n} \int_{E} \exp \left[\left\langle x, e_{k}\right\rangle\left\langle y, e_{k}\right\rangle-\frac{1}{2}\left\langle y, e_{k}\right\rangle^{2}\right] d v(y), \quad n \in \mathbb{N} .
\end{aligned}
$$

Define

$$
X_{k}=\int_{E} \exp \left[\left\langle x, e_{k}\right\rangle\left\langle y, e_{k}\right\rangle-\frac{1}{2}\left\langle y, e_{k}\right\rangle^{2}\right] d v(y)-1, \quad k \in \mathbb{N} .
$$

Then $\left\{X_{k}\right\}$ is an independent random sequence on $(E, \mu)$ such that $\mathbb{E}_{x}\left[X_{k}(x)\right]=0$ and $1+X_{k}>0$, a.s. $(\mu), k \in \mathbb{N}$. Therefore if we prove that 
$\prod_{k=1}^{\infty}\left(1+X_{k}\right)>0$, a.s. $(\mu)$, then by Lemma $7 \phi_{n}=\prod_{k=1}^{n}\left(1+X_{k}\right)$ converges in $L^{1}(\mu)$. By Lemma 8 it is enough to prove the convergences of $\sum_{k} X_{k}$ and $\sum_{k} X_{k}^{2}$.

In fact we have by the symmetricity of $\left\{\left\langle y, e_{k}\right\rangle\right\}$

$$
\begin{aligned}
X_{k}= & \int_{E} \sum_{n=0}^{\infty} \frac{1}{n !}\left\langle x, e_{k}\right\rangle^{n}\left\langle y, e_{k}\right\rangle^{n} e^{-(1 / 2)\left\langle y, e_{k}\right\rangle^{2}} d v(y)-1 \\
= & \sum_{n=0}^{\infty} \frac{1}{(2 n) !}\left\langle x, e_{k}\right\rangle^{2 n} \int_{E}\left\langle y, e_{k}\right\rangle^{2 n} e^{-(1 / 2)\left\langle y, e_{k}\right\rangle^{2}} d v(y) \\
& -\sum_{n=0}^{\infty} \int_{E} \frac{1}{n !} \frac{1}{2^{2 n}}\left\langle y, e_{k}\right\rangle^{2 n} e^{-(1 / 2)\left\langle y, e_{k}\right\rangle^{2}} d v(y) \\
= & \frac{1}{2}\left\{\left\langle x, c_{k}\right\rangle^{2}-1\right\} \int_{E}\left\langle y, e_{k}\right\rangle^{2} e^{-(1 / 2)\left\langle y, e_{k}\right\rangle^{2}} d v(y) \\
& +\sum_{n=2}^{\infty}\left\{\frac{\left\langle x, e_{k}\right\rangle^{2 n}}{(2 n) !}-\frac{1}{2^{n} n !}\right\} \int_{E}\left\langle y, e_{k}\right\rangle^{2 n} e^{-(1 / 2)\left\langle y, e_{k}\right\rangle^{2}} d v(y) \\
= & V_{k}+W_{k} .
\end{aligned}
$$

Since $\left\{\left\langle x, e_{k}\right\rangle\right\}$ is a standard Gaussian sequence on $(E, \mu)$ and by Schwarz' inequality we have

$$
\begin{aligned}
\sum_{k}\left|\int_{E}\left\langle y, e_{k}\right\rangle^{2} e^{-(1 / 2)\left\langle y, e_{k}\right\rangle^{2}} d v(y)\right|^{2} \\
\quad \leqslant \sum_{k} \int_{E}\left\langle y, e_{k}\right\rangle^{4} e^{-(1 / 2)\left\langle y, e_{k}\right\rangle^{2}} d v(y) \leqslant 2 M<+\infty,
\end{aligned}
$$

$\sum V_{k}$ and $\sum V_{k}^{2}$ converge almost surely.

On the other hand we have

$$
\begin{aligned}
\sum_{k} \mathbb{E}_{x}\left[\left|W_{k}\right|\right] \leqslant & \sum_{k} \sum_{n \geqslant 2}\left(\frac{1}{(2 n) !} \mathbb{E}\left[\left\langle x, e_{k}\right\rangle^{2 n}\right]+\frac{1}{2^{n} n !}\right) \\
& \times \int_{E}\left\langle y, e_{k}\right\rangle^{2 n} e^{-1 / 2)\left\langle y, e_{k}\right\rangle^{2}} d v(y) \\
= & 2 \sum_{n \geqslant 2} \frac{1}{2^{n} n !} \int_{E}\left\langle y, e_{k}\right\rangle^{2 n} e^{-(1 / 2)\left\langle y, e_{k}\right\rangle^{2}} d v(y) \leqslant 2 M<+\infty .
\end{aligned}
$$

Therefore $\sum W_{k}$ converges absolutely a.s. $(\mu)$ so that $\sum W_{k}^{2}$ converges almost surely.

These imply that $\sum_{k} X_{k}=\sum_{k} V_{k}+\sum_{k} W_{k}$ and $\sum_{k} X_{k}^{2}=\sum_{k} V_{k}^{2}+$ $2 \sum_{k} V_{k} W_{k}+\sum_{k} W_{k}^{2}$ converge almost surely $(\mu)$. 
EXAMPLE 2. Let $E=R^{\mathbb{N}}$, the space of all real sequences, $\mu$ is the product measure of copies of $N(0,1)$. Then we have $H=l_{2}$. Let $v$ be the product measure of $N\left(0, \sqrt{v_{k}}\right), v_{k}>0, k \in \mathbb{N}$. Then $\mu * v \sim \mu$ if $\sum_{k} v_{k}^{2}<+\infty$.

Remark that $v\left(l_{2}\right)=0$ if $\sum v_{k}=+\infty$.

In fact let $\left\{e_{k}\right\}$ be the canonical CONS of $l_{2}$. Then we have for every $k, n \in \mathbb{N}$

$$
\begin{aligned}
& \sum_{n=2}^{\infty} \frac{1}{2^{n} n !} \sum_{k} \int_{E}\left\langle y, e_{k}\right\rangle^{2 n} e^{-(1 / 2)\left\langle y, e_{k}\right\rangle^{2}} d v(y) \\
& \quad=\sum_{k=1}^{\infty}\left\{\left\{e^{(1 / 2)\left\langle y, e_{k}\right\rangle^{2}}-1-\frac{1}{2}\left\langle y, e_{k}\right\rangle^{2}\right\} e^{-(1 / 2)\left\langle y, e_{k}\right\rangle^{2}} d v(y)\right. \\
& \quad=\sum_{k=1}^{\infty}\left\{1-\frac{1}{\sqrt{1+v_{k}}}-\frac{1}{2} \frac{v_{k}}{\sqrt{1+v_{k}}}\right\} \\
& \quad=\sum_{k=1}^{\infty}\left\{\frac{v_{k}^{2}}{2{\sqrt{1+v_{k}}}^{3}} \frac{2 \sqrt{1+v_{k}}+1}{\left(\sqrt{1+v_{k}}+1\right)^{2}}\right\} \leqslant \sum_{k} v_{k}^{2}<+\infty
\end{aligned}
$$

Corollary. Let $v$ be a Radon measure on $E$ and assume that there exists a CONS $\left\{e_{k}\right\}$ of $H$ chosen from $E^{*}$ such that $\left\{\left\langle y, e_{k}\right\rangle\right\}$ is an independent symmetric random sequence on $(E, v)$ and

$$
\int \exp \left[\frac{1}{2}\left(\sum_{k}\left\langle y, e_{k}\right\rangle^{4}\right)^{1 / 2}\right] d v(y)<+\infty
$$

Then $\mu * v \sim \mu$.

Proof. We have

$$
\begin{aligned}
& \sum_{n \geqslant 2} \frac{1}{2^{n} n !} \sum_{k=1}^{\infty} \int\left\langle y, e_{k}\right\rangle^{2 n} e^{-(1 / 2)\left\langle y, e_{k}\right\rangle^{2}} d v(y) \leqslant \sum_{n \geqslant 2} \frac{1}{n !} \int \sum_{k}\left(\frac{\left\langle y, e_{k}\right\rangle^{2}}{2}\right)^{n} d v(y) \\
& \quad \leqslant \sum_{n \geqslant 2} \frac{1}{n !} \int\left\{\sum_{k} \frac{\left\langle y, e_{k}\right\rangle^{4}}{4}\right\}^{n / 2} d v(y) \leqslant \int \exp \left[\frac{1}{2}\left(\sum_{k}\left\langle y, e_{k}\right\rangle^{4}\right)^{1 / 2}\right] d v(y) \\
& \quad<+\infty .
\end{aligned}
$$

EXAMPLE 3. Let $\mu$ be the same as in Example 2 and $v=$ $\prod_{n} \frac{1}{2}\left(\delta_{a_{n}}+\delta_{-a_{n}}\right)$ where $\sum_{n} a_{n}^{4}<+\infty$. Then $\mu * v \sim \mu$.

$v\left(l_{2}\right)=1$ if and only if $\sum_{n} a_{n}^{2}<+\infty$. 


\section{ACKNOWLEDGMENTS}

The author wishes to show his hearty thanks to Professor C. R. Baker, who drew his attention to the problem, Professor J. Bourgain, who gave the proof of Lemma 3, and Professor M. Talagrand for valuable discussions.

\section{REFERENCES}

1. C. R. BAKER, Conditions for absolute continuity, in "Probability in Banach spaces II," Lecture Notes in Mathematics No. 709, pp. 33-44, Springer-Verlag, Berlin/New York, 1979.

2. S. Bochner and K. Chandrasekharan, "Fourier Transforms," Princeton Univ. Press, Princeton, N.J., 1949.

3. C. Borell, Gaussian Radon measures on locally convex spaces, Math. Scand. 38 (1976), 265-284.

4. L. Gross, Measurable functions on Hilbert space, Trans. Amer. Math. Soc. 105 (1962), 372-390.

5. J. NeVEU, "Martingales à temps discret," Masson, Paris, 1972.

6. W. RudiN, "Real and Complex Analysis," McGraw-Hill, New York, 1966.

7. H. Sato and Y. Okazaki, Separabilities of a Gaussian Radon measure, Ann. Inst. $H$. Poincaré 11 (1975), 287-298. 